\title{
A stochastic approach to rainfall forecasting in the space-time domain: the PRAISEST model
}

\author{
B. Sirangelo \& D. L. De Luca \\ Dipartimento di Difesa del Suolo, Università della Calabria, Rende, Italy
}

\begin{abstract}
This paper introduces a new stochastic technique to forecast rainfall in the spacetime domain: the PRAISEST model (Prediction of Rainfall Amount Inside Storm Events: Space and Time). The model is the extension of the previously presented approach to at-site prediction. PRAISEST is based on the assumption that hourly rainfall in a generic point, denoted by $H$, can be predicted, with a certain probability, by means of the stochastic process that takes into account either a variable $Z$, representing antecedent precipitation at the same point, either a variable $W$, representing simultaneous rainfall at neighbour points. The mathematical background is given by a triple power transformation of the Al-Saadi and Young's trivariate probability distribution, which allows to fit the first and second order sample statistics of $H, Z$ and $W$ and the sample correlations values $r_{H W}, r_{H Z}$ and $r_{W Z}$. As a study area, the Calabria region in Southern Italy was selected. The region was discretised by a $10 \mathrm{~km}$ x $10 \mathrm{~km}$ cell grid, according to the hourly raingauge network density in this area. Storm events belonging to the 1990-2004 period were analyzed to test the performances of the PRAISEST model.
\end{abstract}

Keywords: hourly rainfall forecasting, space-time stochastic processes, trivariate probability distributions.

\section{Introduction}

In the present work the forecast model of precipitation named PRAISEST (Prediction of Rainfall Amount Inside Storm Events: Space and Time) is described. To forecast rainfall heights named $H$, in a generic cell of space domain, the existing link among the random variables $H, W$ and $Z$ is used. The variable $Z$ represents antecedent precipitation at the same point and the variable 
$W$ represents simultaneous rainfall at neighbour points. $Z$ describes the temporal memory of the precipitation field. The paper is structured in four paragraphs, excluding the introduction. In Section 2 the theoretical bases of the stochastic model are illustrated, while in Sections 3 and 4, respectively, the techniques of fitting and the algorithm of rain fields generation are shown. Finally, Section 5 concerns the application of the model to the region Calabria, in Southern Italy, opportunely discretized in square cells of resolution equal to $10 \mathrm{~km}$.

\section{The PRAISEST model}

The PRAISEST model constitutes the space-time generalization of the atsite model named PRAISE (Sirangelo and Versace [1]; Sirangelo and Braca [2]). In this context, $H_{i+1}$ is a non-negative variable describing the rainfall height accumulated on an interval $\Delta t$ between the instants $i \Delta t$ and $(i+1) \Delta t$, and on a spatial cell of size $\Delta x \Delta y$. Besides, there are the random variables $Z_{i}^{(v)}$, linear function of the $v$ variables $H_{i}, H_{i-1}, \ldots, H_{i-v+1}$, representing rainfall heights accumulated on intervals between $(i-1) \Delta t$ and $i \Delta t,(i-2) \Delta t$ and $(i-1) \Delta t, \ldots$, $(i-v) \Delta t$ and $(i-v+1) \Delta t$, on the same cell of $H_{i+1}$, and $W$, representing rainfall accumulated on interval $\Delta t$, at neighbour cells.

If $W$ is the simultaneous rainfalls accumulated between $i \Delta t$ and $(i+1) \Delta t$, it is indicated as $W_{i+1}$ and one will talk about Implicit scheme; in the case in which one considers rainfalls accumulated between $(i-1) \Delta t$ and $i \Delta t$, the variable is indicated as $W_{i}$ and the scheme is called Explicit (fig. 1).

In the following, for notation simplicity, the subscripts of random variables $H$, $W$ and $Z$ will be removed where possible.

\subsection{Identification of $Z$ and $W$ variables}

The random variable $Z$ is calculated as linear function of the $v$ rainfall heights $H_{i-v+1}, \ldots H_{i-1}, H_{i}$ referred to the reference cell:

$$
Z_{i}^{(v)}=\sum_{j=0}^{v-1} \alpha_{j} H_{i-j}
$$

with $0 \leq \alpha_{j} \leq 1, j=0,1, \ldots, v-1$ and $\sum_{j=0}^{v-1} \alpha_{j}=1$. The coefficients $\alpha_{j}$ are evaluated with a technique of linear filtering (De Luca [3]) and the adopted criterion is the maximization of the linear correlation coefficient $\rho_{H Z}$. The random variable $W$ represents a weighed average of the rainfall heights in the four adjacent cells, referred to the time forecast interval (Implicit scheme) or to the previous interval (Explicit scheme). 


$$
W_{i+1}=\sum_{j=1}^{4} \beta_{j} H_{i+1}^{(j)}
$$

$$
W_{i}=\sum_{j=1}^{4} \beta_{j} H_{i}^{(j)}
$$

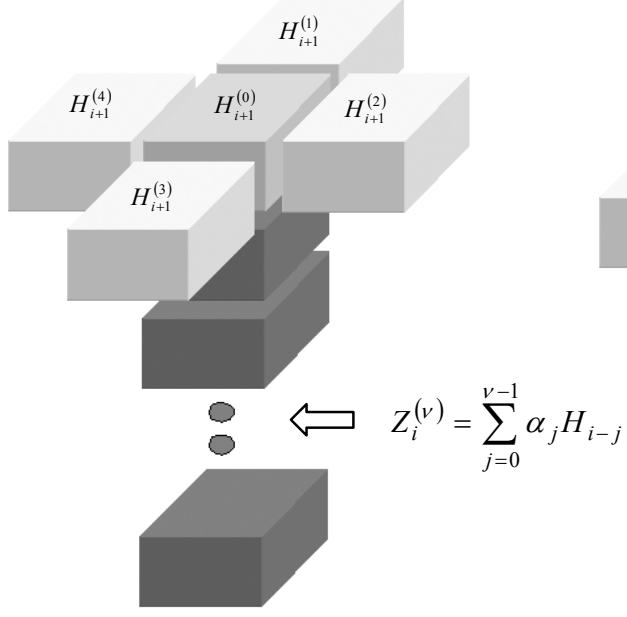

a)
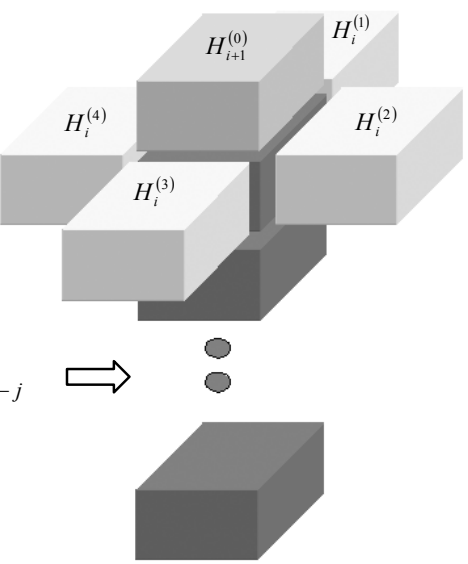

b)

Figure 1: a) Implicit Scheme; b) Explicit scheme.

The expressions of $W$ for the 2 cases are:

$$
W_{i+1}=\sum_{j=1}^{4} \beta_{j} H_{i+1}^{(j)} \quad \text { and } \quad W_{i}=\sum_{j=1}^{4} \beta_{j} H_{i}^{(j)}
$$

where, indicated with $\rho_{1,0}, \rho_{2,0}, \rho_{3,0}, \rho_{4,0}$ the linear correlation coefficients between the reference cell 0 and the neighbour cells $1,2,3$ and 4 (fig. 1), the coefficients $\beta_{j}$ can be set equal to:

$$
\beta_{j}=\frac{\rho_{j, 0}}{\sum_{j=1}^{4} \rho_{j, 0}} \quad j=1,2,3,4
$$

It is clearly that if the field can be considered as locally isotropic, the coefficients $\beta_{j}$ assume the same value equal value to $1 / 4$.

\subsection{Structure of the joined probability density}

As regards the joined probability density $f_{H, W, Z}(h, w, z)$ it is necessary to consider the mixed nature of random variables $H, W$ and $Z$. The non-negative 
variable $H$ is characterized by a finite probability in correspondence of the null value and by infinitesimal probabilities in correspondence of the positive values. Such considerations hold also for the variables $W$ and $Z$. Then, indicated with $p_{+,+,+}, p_{+,+, \bullet}, p_{+, \bullet,+}, p_{\bullet,+,+}, p_{+, \bullet, \bullet}, p_{\bullet,+, \bullet}, p_{\bullet, \bullet,+}$ e $p_{\bullet \bullet \bullet \bullet \bullet \bullet}$ the probabilities associated to the events $H>0 \cap W>0 \cap Z>0, H>0 \cap W>0 \cap Z=0$, $H>0 \cap W=0 \cap Z>0, \quad H=0 \cap W>0 \cap Z>0, \quad H>0 \cap W=0 \cap Z=0$, $H=0 \cap W>0 \cap Z=0, \quad H=0 \cap W=0 \cap Z>0$ e $H=0 \cap W=0 \cap Z=0$, the joined probability density $f_{H, W, Z}(h, w, z)$ assumes the form:

$$
\begin{aligned}
f_{H, W, Z}(h, w, z)= & p_{\bullet, \bullet \bullet} \delta(h) \delta(w) \delta(z)+p_{+, \bullet, \bullet} \cdot f_{H, 0,0}^{(+, \bullet, \bullet)}(h) \cdot \delta(w) \delta(z)+ \\
& p_{\bullet,+, \bullet} \cdot f_{0, W, 0}^{(\bullet,+, \bullet)}(w) \cdot \delta(h) \delta(z)+p_{\bullet, \bullet,+} \cdot f_{0,0, Z}^{(\bullet \bullet \bullet,+)}(z) \delta(h) \delta(w)+ \\
& p_{+,+, \bullet} \cdot f_{H, W, 0}^{(+,+, \bullet)}(h, w) \delta(z)+p_{+, \bullet,+} \cdot f_{H, 0, Z}^{(+, \bullet,+}(h, z) \delta(w)+ \\
& p_{\bullet,+,+} \cdot f_{0, W, Z}^{(\bullet,+,+)}(w, z) \delta(h)+p_{+,+,+} \cdot f_{H, W, Z}^{(+,+,+)}(h, w, z)
\end{aligned}
$$

where the symbol $\delta(\cdot)$ indicates the Dirac's delta and, clearly, $p_{\bullet, \bullet \bullet}+p_{+, \bullet, \bullet}+p_{\bullet,+, \bullet}+p_{\bullet, \bullet,+}+p_{+,+, \bullet}+p_{+, \bullet,+}+p_{\bullet,+,+}+p_{+,+,+}=1$.

In the model here illustrated, the analytical expression of the density $f_{H, W, Z}^{(+,+,)}(h, w, z)$ is derived from a triple power transformation of the Al-Saadi and Young's [4] trivariate exponential distribution, obtaining:

$$
\begin{aligned}
& f_{H, S, Z}^{(+,+,+)}(h, s, z)=\alpha_{H}^{(+,+,+)} \beta_{H}^{(+,+,+)} h^{\beta_{H}^{(+,+,+}-1} \alpha_{W}^{(+,+,+)} \beta_{W}^{(+,+,+)} w^{\beta_{W}^{(+,++)}-1} . \\
& \alpha_{Z}^{(+,+,+)} \beta_{Z}^{(+,+,+)} z^{\beta_{Z}^{(+,++)}-1}\left(\theta^{(+,+,+)}\right)^{2} . \\
& \exp \left[-\theta^{(+,+,+)}\left(\alpha_{H}^{(+,+,+)} h^{\beta_{H}^{(+,+,)}}+\alpha_{W}^{(+,+,+)} w^{\beta_{W}^{(+,+,+}}+\alpha_{Z}^{(+,+,+)} z^{\beta_{Z}^{(+,+,+}}\right)\right] . \\
& \sum_{i=0}^{\infty} \frac{1}{(i !)^{3}}\left[\left(\theta^{(+,+,+)}\right)^{2}\left(\theta^{(+,+,+)}-1\right)\left(\alpha_{H}^{(+,+,+)} h^{\beta_{H}^{(+,++}}\right)\left(\alpha_{W}^{(+,+,+)} w^{\beta_{W}^{(+,++}}\right)\left(\alpha_{Z}^{(+,+,+)} z^{\left.\beta_{Z}^{(+,++}\right)}\right)\right]^{i}
\end{aligned}
$$

with $\alpha_{H}^{(+,+,+)}>0, \beta_{H}^{(+,+,+)}>0, \alpha_{W}^{(+,+,+)}>0, \beta_{W}^{(+,+,+)}>0, \alpha_{Z}^{(+,+,+)}>0, \beta_{Z}^{(+,+,+)}>0$ and $\theta^{(+,+,+)} \geq 1$.

Higher values of $\theta^{(+,+,+)}$give higher correlation values among $H, W$ and $Z$ variables.

Referring to the mathematical expressions of the remaining densities appearing in eqn (3), Weibull-Bessel laws (Sirangelo and Versace, [1]): 


$$
\begin{aligned}
& f_{H, W, 0}^{(+,+, \bullet}(h, w)= \theta^{(+,+, \bullet)} \alpha_{H}^{(+,+, \bullet)} \beta_{H}^{(+,+, \bullet)}(h)_{H}^{\beta_{H}^{(+,+\bullet}-1} \alpha_{W}^{(+,+, \bullet)} \beta_{W}^{(+,+, \bullet)}(w)^{\beta_{W}^{(+,+\bullet)}-1} . \\
& \exp \left\{-\theta^{(+,+, \bullet)}\left[\alpha_{H}^{(+,+, \bullet)}(h)_{H}^{\beta_{H}^{(+,+\bullet)}}+\alpha_{W}^{(+,+, \bullet)}(w)_{W}^{\beta_{W}^{(+,+\bullet}}\right]\right\} . \\
& \mathrm{I}_{0}\left[2 \sqrt{\theta^{(+,+, \bullet)}\left(\theta^{(+,+, \bullet)}-1\right) \alpha_{H}^{(+,+, \bullet)}(h)^{\beta_{H}^{(+,+\bullet}} \alpha_{W}^{(+,+, \bullet)}(w)^{\beta_{W}^{(+,+\bullet}}}\right]
\end{aligned}
$$

with $h>0, w>0 ; \alpha_{H}^{(+,+, \bullet)}>0, \beta_{H}^{(+,+, \bullet)}>0, \alpha_{W}^{(+,+, \bullet)}>0, \beta_{W}^{(+,+, \bullet)}>0, \theta^{(+,+, \bullet)} \geq 1$;

$$
\begin{aligned}
f_{H, 0, Z}^{(+, \bullet,+)}(h, z)= & \theta^{(+, \bullet,+)} \alpha_{H}^{(+, \bullet,+)} \beta_{H}^{(+, \bullet,+)}(h)_{H}^{\beta_{H}^{(+, \bullet,+)}-1} \alpha_{Z}^{(+, \bullet,+)} \beta_{Z}^{(+, \bullet,+)}(z)^{\beta_{Z}^{(+, \bullet,+)}-1} \\
& \exp \left\{-\theta^{(+, \bullet,+)}\left[\alpha_{H}^{(+, \bullet,+)}(h)_{H}^{\beta_{H}^{(+, \bullet+)}}+\alpha_{Z}^{(+, \bullet,+)}(z)^{\beta_{Z}^{(+, \bullet+}}\right]\right\} \\
& \mathrm{I}_{0}\left[2 \sqrt{\theta^{(+, \bullet,+)}\left(\theta^{(+, \bullet,+)}-1\right) \alpha_{H}^{(+, \bullet,+)}(h)^{\beta_{H}^{(+, \bullet+)}} \alpha_{Z}^{(+, \bullet,+)}(z)^{\beta_{Z}^{(+, \bullet+)}}}\right]
\end{aligned}
$$

with $h>0, z>0 ; \alpha_{H}^{(+, \bullet,+)}>0, \beta_{H}^{(+, \bullet,+)}>0, \alpha_{Z}^{(+, \bullet,+)}>0, \beta_{Z}^{(+, \bullet,+)}>0, \theta^{(+, \bullet,+)} \geq 1$;

$$
\begin{aligned}
f_{0, W, Z}^{(\bullet,+,+}(w, z)= & \theta^{(\bullet,+,+)} \alpha_{W}^{(\bullet,+,+)} \beta_{W}^{(\bullet,+,+)}(w)^{\beta_{H}^{(\bullet,++)}-1} \alpha_{Z}^{(\bullet,+,+)} \beta_{z}^{(\bullet,+,+)}(z)^{\beta_{z}^{(\bullet,+,+}-1} \\
& \exp \left\{-\theta^{(\bullet,+,+)}\left[\alpha_{W}^{(\bullet,+,+)}(w)^{\beta_{W}^{(\bullet,+,)}}+\alpha_{Z}^{(\bullet,+,+)}(z)^{\beta_{z}^{(\bullet,+,+}}\right]\right\} \\
& \mathrm{I}_{0}\left[2 \sqrt{\theta^{(\bullet,+,+)}\left(\theta^{(\bullet,+,+)}-1\right) \alpha_{W}^{(\bullet,+,+)}(w)^{\beta_{W}^{(\bullet,++)}} \alpha_{Z}^{(\bullet,+,+)}(z)^{\beta_{z}^{(\bullet,+,)}}}\right]
\end{aligned}
$$

with $w>0, z>0 ; \alpha_{W}^{(\bullet,+,+)}>0, \beta_{W}^{(\bullet,+,+)}>0, \alpha_{Z}^{(\bullet,+,+)}>0, \beta_{z}^{(\bullet,+,+)}>0, \theta^{(\bullet,+,+)} \geq 1$; and Weibull laws:

$$
f_{H, 0,0}^{(+, \bullet, \bullet)}(h)=\alpha_{H}^{(+, \bullet, \bullet)} \beta_{H}^{(+, \bullet, \bullet)} h^{\beta_{H}^{(+, \bullet \bullet \bullet}-1} \exp \left[\alpha_{H}^{(+, \bullet, \bullet)} h^{\beta_{H}^{(+, \bullet \bullet \bullet}}\right]
$$

with $h>0 ; \alpha_{H}^{(+, \bullet, \bullet)}>0 ; \beta_{H}^{(+, \bullet, \bullet)}>0$;

$$
f_{0, W, 0}^{(\bullet,+,)}(w)=\alpha_{W}^{(\bullet,+, \bullet)} \beta_{W}^{(\bullet,+, \bullet)} w^{\beta_{W}^{(\bullet,+, \bullet)}-1} \exp \left[\alpha_{W}^{(\bullet,+, \bullet)} h^{\beta_{W}^{(\bullet,+, \bullet)}}\right]
$$

with $w>0 ; \alpha_{W}^{(\bullet,+, \bullet)}>0 ; \beta_{W}^{(\bullet,+, \bullet)}>0$;

$$
f_{0,0, Z}^{(\bullet, \bullet,+)}(z)=\alpha_{Z}^{(\bullet, \bullet,+)} \beta_{Z}^{(\bullet, \bullet,+)} z^{\beta_{Z}^{(\bullet, \bullet,+)}-1} \exp \left[\alpha_{Z}^{(\bullet, \bullet,+)} \beta^{\beta_{Z}^{(\bullet, \bullet,+)}}\right]
$$

with $z>0 ; \alpha_{Z}^{(\bullet, \bullet,+)}>0 ; \beta_{Z}^{(\bullet, \bullet,+)}>0$;

are adopted. In the eqns $(6 \mathrm{a}-\mathrm{c}), I_{0}(\cdot)$ is the modified Bessel function of zero order (Abramowitz and Stegun [5]). 


\section{Calibration of the model}

The trivariate probability distribution function $f_{H, W, Z}(h, w, z)$ presents 35 parameters, for a generic cell, and the model could appear overparameterized. However, if it is considered, as an example, an application to fields of precipitation cumulated to hour scale, having a sampling of at least 10 years for a generic rain gauge, for a total of 87600 data/rain gauge, it can be noted that the ratio data/parameters is approximately equal to 2500; such ratio, moreover, remains equal to 500 considering only positive rainfall heights.

The parametric estimate has been made using the method of the moments. First of all, starting from the samples $\left[h_{1}, h_{2}, \ldots, h_{N}\right],\left[w_{1}, w_{2}, \ldots, w_{N}\right]$, $\left[z_{1}, z_{2}, \ldots, z_{N}\right]$, for every event defined at paragraph 2.2 , the correspondent probability distribution parameters, are estimated. In particular, in the case of the event $H>0 \cap W>0 \cap Z>0$, as regards the parameter $\theta^{(+,+,+)}$, the estimation is performed minimizing the following function:

$$
\begin{aligned}
& R\left(\theta^{(+,+,+)}\right)= \\
& \quad \omega_{1}\left(\rho_{H W}^{(+,+,+)}-r_{H W}^{(+,+,+)}\right)^{2}+\omega_{2}\left(\rho_{H Z}^{(+,+,+)}-r_{H Z}^{(+,+,+)}\right)^{2}+\omega_{3}\left(\rho_{W Z}^{(+,+,+}-r_{W Z}^{(+,+,+)}\right)^{2}
\end{aligned}
$$

where $r_{H W}^{(+,+,+)}, r_{H Z}^{(+,+,+)}, r_{W Z}^{(+,+,+)}$are the sample linear correlation coefficients, $\rho_{H W}^{(+,+,+)}, \rho_{H Z}^{(+,+,+)}, \rho_{W Z}^{(+,+,+)}$are the theoretical ones, and the sum of the weights $\omega_{1}, \omega_{2}$ and $\omega_{3}$ is unitary. The function (8) depends only on the parameter $\theta^{(+,+,+)}$, since the remaining parameters are already evaluated by the classic expression of the Weibull distribution parameter estimation.

\section{Rain fields generation algorithms}

In real-time applications of the Explicit scheme of PRAISEST model, either Z's value either $W$ 's one are known, so it is possible to generate the value of the variable $H$ on the whole domain, according to the usual Monte Carlo techniques. In every cell, rainfall heights are generated by means of the cumulate distribution function $F\left(H_{i+1} \leq h_{i+1} \mid W_{i}=w_{i}, Z_{i}=z_{i}\right)$, clearly with opportune expressions in the four possible cases: $W_{i}$ null or positive and $Z_{i}$ null or positive.

On the contrary, when Implicit scheme is used, the generation of the rainfall heights on the entire domain differs from the standard Monte Carlo approach. In fact, at the current time $i$, the values of the random variable $Z_{i}$ in every cell are known, but values of $W_{i+1}$ and $H_{i+1}$ on the entire domain must be generated. Such generation cannot be independently carried out because the variables are linked by congruence equations. This problem can be solved using the following algorithms (fig. 2): 
1. The starting cell 0 is chosen in random way, and, knowing the value of $Z_{i}$, generation is made using the random number $R_{U}^{(0)}$, by the formula $h_{i+1}^{(0)}=F^{-1}\left(R_{U}^{(0)} \mid W_{i+1}^{(0)} \geq 0, Z_{i}^{(0)}=z_{i}^{(0)}\right)$, i.e. the variable $H_{i+1}$ is generated supposing zero as lower bound for $W_{i+1}$;

2. Considering the cells near to cell 0 , numbered as $1,2,3$ and 4 (fig. 2), for everyone the only known rainfall height, at the adjacent cells, is $h_{i+1}^{(0)}$. This value, multiplied for the corresponding weight $\beta$, constitutes the lower bound for the variable $W_{i+1}^{(j)}, j=1, \ldots, 4$.

Random numbers $R_{U}^{(j)}, j=1,2,3,4$, are generated and, then, the formula $h_{i+1}^{(j)}=F^{-1}\left(R_{U}^{(j)} \mid W_{i+1}^{(j)} \geq 0.25 \cdot h_{i+1}^{(0)}, Z_{i}^{(j)}=z_{i}^{(j)}\right)$ is used;
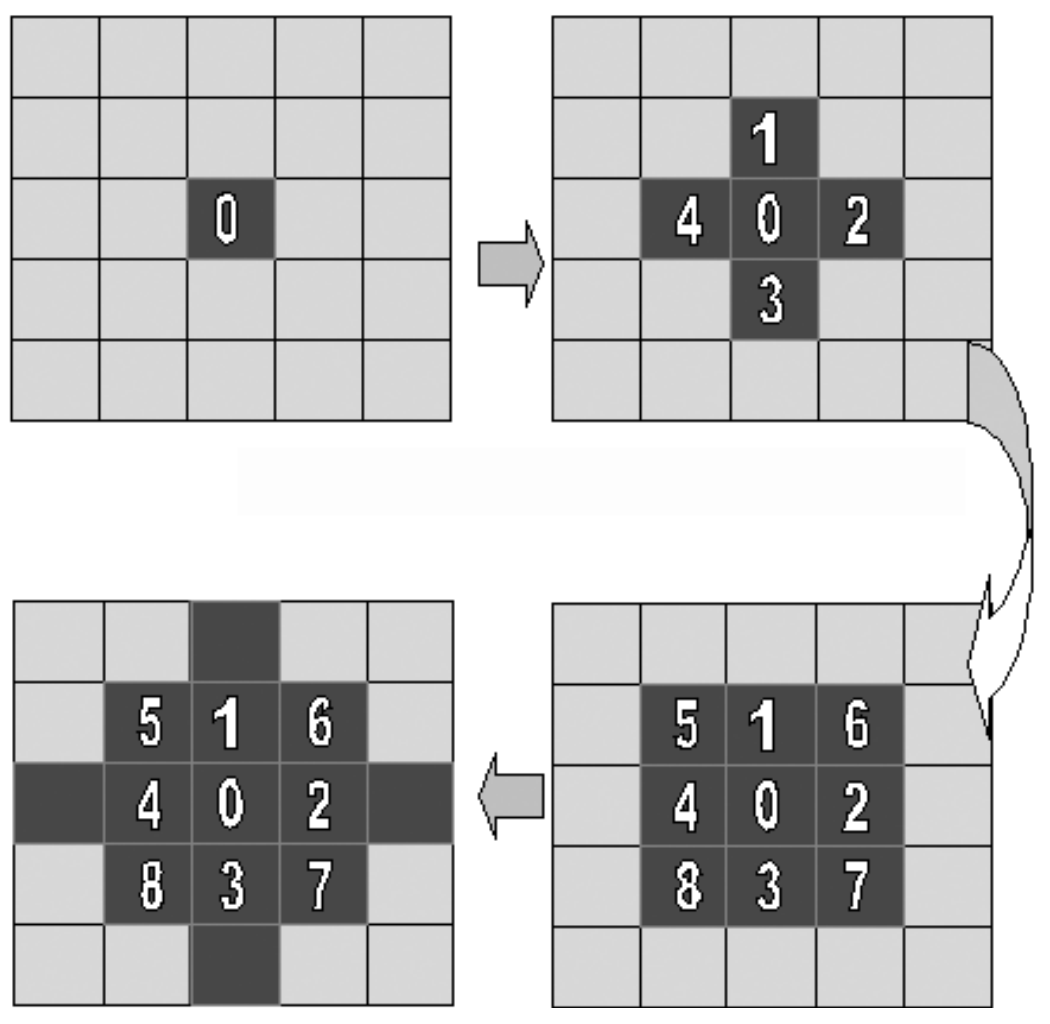

Figure 2: $\quad$ Rain fields generation algorithm for the Implicit scheme. 
3. The successive cells are considered and the lower bound of $W_{i+1}^{(\cdot)}$ is set equal to the weighed sum of already simulated heights of the neighbour cells.

As in the Explicit scheme, the function $F(H \leq h \mid W \geq w, Z=z)$ differs in the four possible cases: $W_{i+1}$ 's lower bound null or positive and $Z_{i}$ null or positive.

\section{Application}

Model calibration was performed using the hourly rain heights database of raingauge network of "Centro Funzionale Meteorologico Idrografico Mareografico ARPACAL". The raingauge network, located in Calabria and Basilicata regions, Southern Italy, is composed by 92 stations for the period 1990-2001, and by 126 stations from 2002 (fig. 3). Approximately, 14 million of hourly rainfalls form the database, of which about $7 \%$ are rainy.

The region was discretized by $10 \mathrm{~km} \mathrm{x} 10 \mathrm{~km}$ cell grid, according to the hourly raingauge network density in this area.

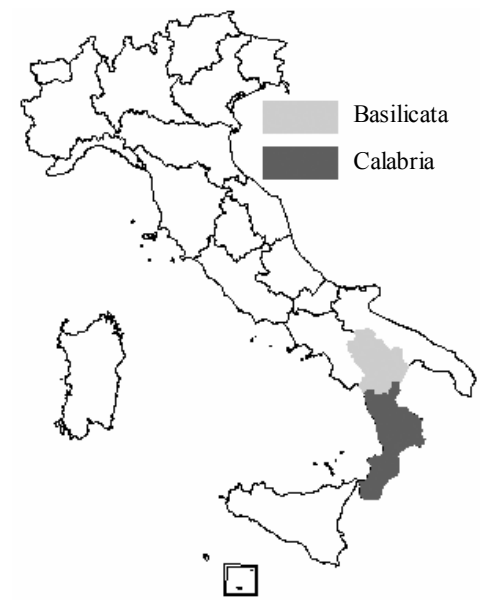

a)

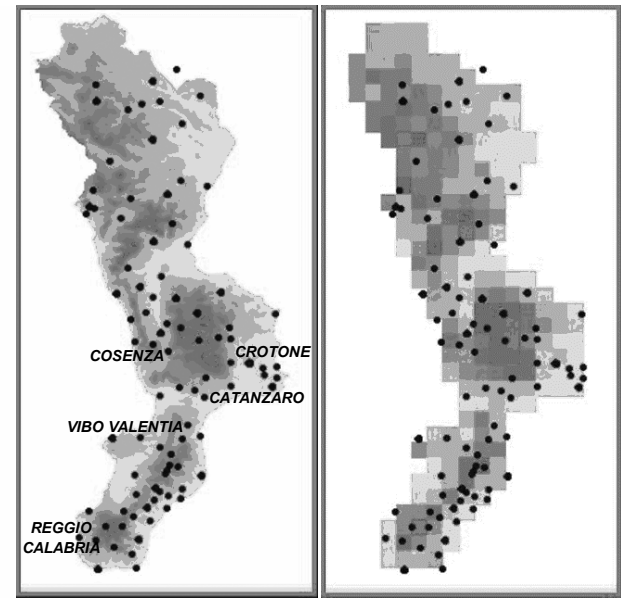

b)

c)

Figure 3: a) Location of Basilicata and Calabria regions; b) Rainguage network; c) Discretization of spatial domain.

Having historical series not on a regular mesh, the model parameters have been estimated for every rain gauge. Subsequently, each parameter was mapped on the discretized domain by using a spline technique. The analysis has shown a constant value for the parameter $v$, equal to 8 . Moreover, the hypothesis of locally isotropic field cannot be rejected and, then, $\beta_{j}=0.25, j=1,2,3,4$, for every cell, have been assumed. A set of estimated parameters is reported in the table 1 . 
Table 1: $\quad$ Example of estimated parameters set.

\begin{tabular}{|c|c|c|c|c|c|c|c|c|}
\hline & $\begin{array}{l}p \\
(-)\end{array}$ & $\begin{array}{l}1 / \alpha_{H} \\
(\mathrm{~mm})\end{array}$ & $\begin{array}{l}\beta_{H} \\
(-)\end{array}$ & $\begin{array}{l}1 / \alpha_{W} \\
(\mathrm{~mm})\end{array}$ & $\begin{array}{c}\beta_{W} \\
(-)\end{array}$ & $\begin{array}{l}1 / \alpha_{Z} \\
(\mathrm{~mm})\end{array}$ & $\begin{array}{r}\beta_{Z} \\
(-)\end{array}$ & $\begin{array}{l}\theta \\
(-)\end{array}$ \\
\hline$(+,+,+)$ & 0.062 & 1.27 & 0.76 & 1.25 & 0.81 & 1.10 & 0.75 & 1.94 \\
\hline$(+,+, \bullet)$ & 0.008 & 0.81 & 0.66 & 0.83 & 0.73 & & & 2.12 \\
\hline$(+, \bullet,+)$ & 0.013 & 0.61 & 0.66 & & & 0.61 & 0.65 & 1.75 \\
\hline$(\bullet,+,+)$ & 0.034 & & & 0.46 & 0.64 & 0.44 & 0.56 & 1.16 \\
\hline$(+, \bullet, \bullet)$ & 0.005 & 0.46 & 0.63 & & & & & \\
\hline$(\bullet,+, \bullet)$ & 0.028 & & & 0.45 & 0.59 & & & \\
\hline$(\bullet, \bullet,+)$ & 0.107 & & & & & 0.26 & 0.46 & \\
\hline
\end{tabular}

An example of parameter mapping, referred to $\theta^{(+,+,+)}$, for $H>0 \cap W>0 \cap Z>0$ event, and for the Implicit scheme, is depicted in fig. 4, where only Calabria region is considered.
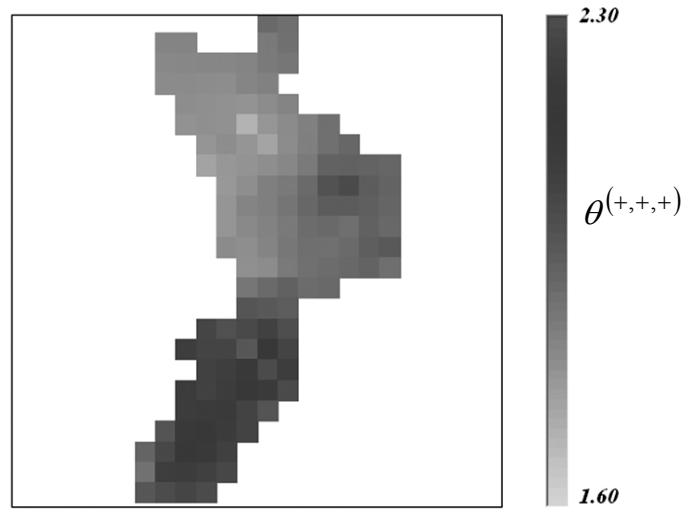

Figure 4: $\quad$ Mapping of the parameter $\theta^{(+,+,+)}$for the Implicit scheme.

The figure shows greater values located in the Southern Calabria, where the variables $H, W$ and $Z$ appear more strongly correlated.

Each rain field simulation requires the knowledge of the rainfalls relative to the eight previous hours. Starting from these, rain fields simulations can be carried out for the successive hours. The temporal extension of the forecast should not exceed six hours. Beyond this limit the results become similar to the unconditional ones and, then, a model update by observed rain fields is necessary. In the application here described, the simulations are carried out repeating the process 1000 times in order to have a large synthetic sample.

In this paper the applications relative to November $24^{\text {th }} 1999$, in the Calabria region, are illustrated. As model memory, the rain fields from 9 p.m. of November $23^{\text {rd }}$ to 4.00 a.m. of November $24^{\text {th }}$ are used. The simulation period starts from 5.00 am and finishes at 10.00 a.m. of November $24^{\text {th }}$. 
In the graphs of fig. 5 and fig. 6, PRAISEST simulation results are depicted, respectively for the Implicit and Explicit schemes.
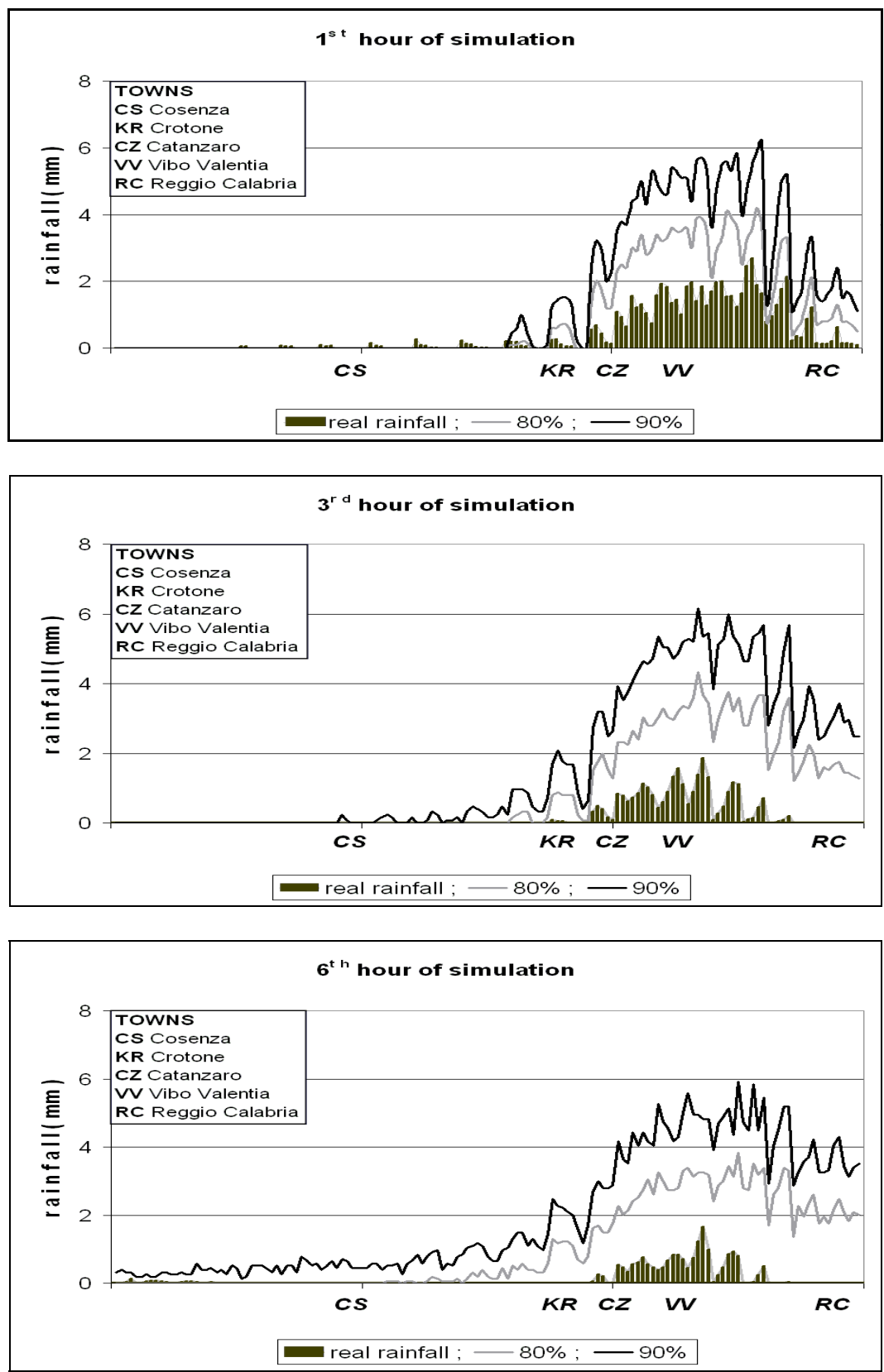

Figure 5: Implicit Scheme: $1^{\text {st }}, 3^{\text {rd }}$ and $6^{\text {th }}$ hour of simulation. 

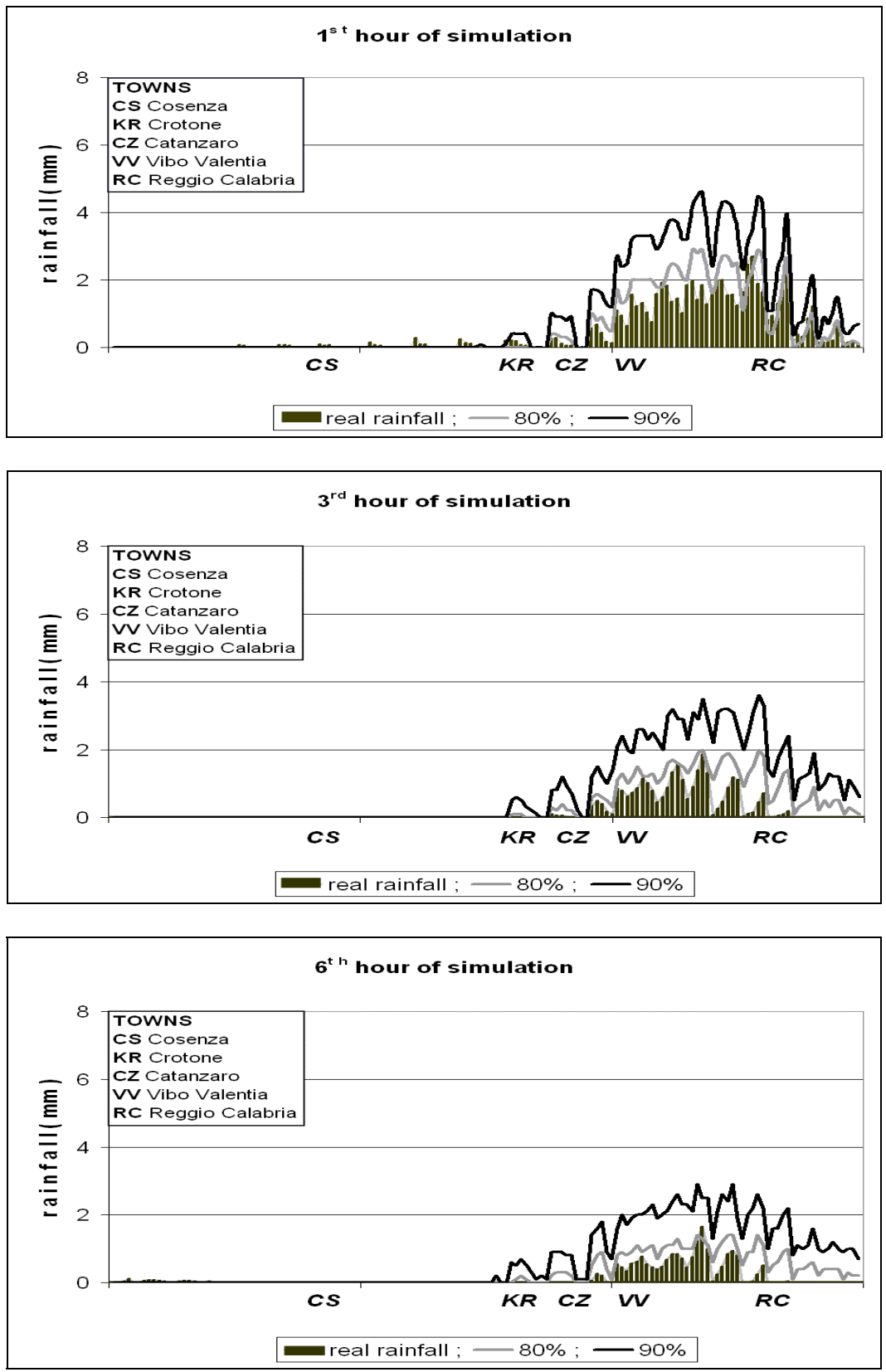

Figure 6: Explicit Scheme. $1^{\text {st }}, 3^{\text {rd }}$ and $6^{\text {th }}$ hour of simulation.

On the abscissa, the cells are sorted from left to right, beginning from North. In the figures, besides the rain histograms effectively occurred, for every cell, 
percentiles $80 \%$ and $90 \%$ of the simulated fields are reported. Following the axis of the abscissas, the towns of Cosenza (CS), Crotone (KR), Catanzaro (CZ), Vibo Valentia (VV) and Reggio Calabria (RC) are met in this order. The diagrams show that the Implicit scheme gives a persistence of the percentiles values, when temporal forecast extension increases. On the contrary, the Explicit scheme exhibits a significant reduction of these percentiles values.

The rainfall heights of the real event, in the six hours of forecast, are clearly inferior to percentiles $80 \%$ in the implicit scheme, while in explicit one the occurred precipitations fall between percentiles $80 \%$ and $90 \%$.

However, it must be stressed that the substantial difference between the two schemes regards the reproduction of the spatial correlative structure of the rain event. As displayed in fig. 7, the effective correlation between $W_{i+1}$ and $H_{i+1}$ is preserved only by the Implicit scheme. The explicit scheme gives lower correlations $\rho_{H_{i+1} W_{i+1}}$ because it only reproduces the correlative structure between $W_{i}$ and $H_{i+1}$.

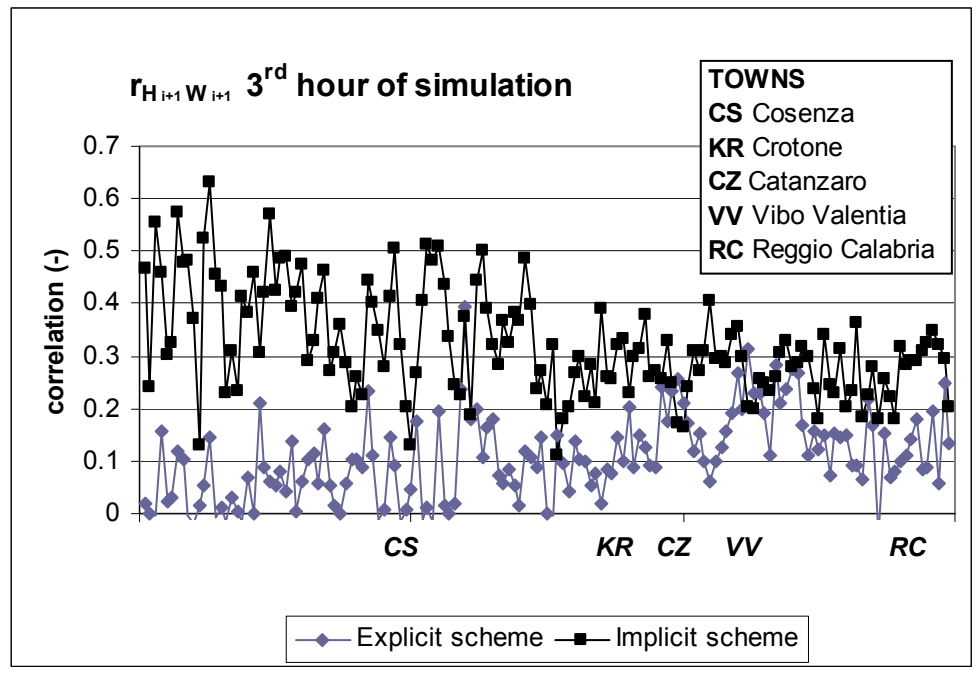

Figure 7: Simultaneous correlation structure for the $3^{\text {rd }}$ hour of simulation.

\section{Conclusion}

In the paper presented herein a space-time model for forecasting rainfall fields named PRAISEST was suggested. Mathematical background is characterized by a trivariate probability distribution, referred to the random variables $H, \mathrm{Z}$ and $W$, representing rainfall in the generic cell, antecedent precipitation in the same cell and rainfall in the adjacent cells. Two different approaches for the PRAISEST model can be used, called Implicit and Explicit schemes. The former one models $W$ at the forecast time, while the second one models $W$ at the current time. 
Because of the high number of parameters in the adopted trivariate distribution, in order to obtain a reliable estimation, it is strictly necessary the availability of a large size sample. In the calibration here presented an hourly rainfall database of fourteen years on over 100 raingauges was used.

The PRAISEST model was applied to simulate the rainfall event of November $24^{\text {th }}, 1999$, in Calabria region, Southern Italy. The results obtained indicate the capability of the model to identify, for the forecast hours, statistical confidence bands containing the real rainfall heights.

The simulation shows that Explicit scheme can be used only for a "quick and dirty" elaboration, while, particularly if there is interest to reproduce the correlative structure of the precipitation field, the Implicit scheme should be preferred, even if it requires a more complex generation algorithm.

\section{References}

[1] Sirangelo B. \& Versace P. Un modello probabilistico per la predizione in tempo reale delle altezze di precipitazione a scala oraria. Proc. XXVIII Convegno di Idraulica e Costruzioni Idrauliche, Potenza, vol. 2, pp. 395414, 2002.

[2] Sirangelo B. \& Braca G. Identification of hazard conditions for mudflow occurrence by hydrological model. Application of FLaIR model to Sarno warning system. Engineering Geology, vol. 73, no. 3-4, pp. 267-276, 2004.

[3] De Luca D.L., Metodi di previsione dei campi di pioggia. Tesi di Dottorato di Ricerca, Università della Calabria, 2005.

[4] Al-Saadi S.D. \& Young D.H. A test for independence in a multivariate exponential distribution with equal correlation coefficient. Journal of Statistical Computation and Simulation, vol. 14, pp. 219-227, 1982.

[5] Abramowitz M. \& Stegun I.A. Handbook of mathematical functions. Dover, New York, 1970. 